\title{
Levels of perceptual processing as a function of stimulus material and spatial location
}

\author{
GIDEON KEREN \\ University of Illinois, Champaign, Illinois 61820
}

\begin{abstract}
Craik and Lockhart (1972) have proposed a framework for human memory research which is based on levels of processing. Most of the studies which support this approach have been concerned with comparing structural vs. semantic tasks. The present paper extends the levels of processing approach to earlier stages of processing and emphasizes levels of perceptual processing. It is suggested that depth of perceptual processing depends on the nature of the stimulus material. Neisser's (1967) theory of preattentive mechanisms and focal attention is shown to be a theory of levels of perceptual processing. Differences in the nature in which these two processes operates are discussed.
\end{abstract}

Craik and Lockhart (1972) have proposed a framework for memory research and have argued "that the memory trace can be understood as a byproduct of perceptual analysis and that the trace persistence is a positive function of the depth to which the stimulus has been analyzed" (Craik \& Lockhart, 1972; p. 671). Most of the evidence in support of this "levels of processing" theory comes from experiments which have used an incidental learning paradigm (Craik \& Tulving, 1975; Hyde \& Jenkins, 1969; Johnston \& Jenkins, 1971; Tresselt \& Mayzner, 1960). Although Craik and Lockhart stress the perceptual nature of the "depth" continuum, most of the supporting studies have used tasks involving structural analyses (number of letters or syllables in a word, sound of words) as opposed to semantic analyses (meaning of the word). These studies have shown that the memory trace under the latter conditions has, indeed, been superior to the former conditions. The present experiment was conducted in order to further extend the existing evidence by utilizing tasks which require levels of perceptual processing.

Neisser (1967) has proposed a theory which assumes "levels" of perceptual processing, namely, preattentive processes and focal attention. Neisser has argued for the existence of these two levels of analysis: "The preattentive mechanisms which form segregated objects and help to direct further processing, and the act of focal attention, which makes more sophisticated analyses of the chosen object" (Neisser, 1967). Briefly, preattentive mechanisms operate at a preliminary stage with rapid and relatively inaccurate processing, which involves detection of physical features, grouping, and localizing the whole percept, and monitoring for critical features

The author would like to acknowledge the help and support of C. W. Eriksen. This study was partly supported by Public Health Service Research Grant MH-1206 to C. W. Eriksen. Thanks are also due to Edward Lichtenstein for many useful comments on earlier drafts of this paper. Requests for reprints should be sent to Gideon Keren, Department of Psychology, University of Southern California, University Park, Los Angeles, California 90007. which might require redirection of focal attention. The subsequent stage of focal attention is said to involve additional analysis, a type of processing which is slower but provides more detailed information and additional elaboration.

What are the conditions under which each of these two processes will operate? The answer to this question is not completely clear. Keren (1975) suggested that these processes were related to a distinction between two kinds of stimulus material, first proposed by Broadbent $(1970,1971)$. One type of stimulus array, labeled "stimulus set," permits selection among its elements on the basis of physical features which are inherent in the stimulus (e.g., selecting a red letter from an array of red and black letters). Elements within the other type of stimulus material, labeled "response set," can not be distinguished solely on the basis of physical features, but rather on the basis of meaning (or additional information) that is conveyed by the stimulus (e.g., selecting a particular digit from an array of digits and letters). Keren (1975) suggested that stimulus set material may be handled by preattentive mechanisms, while response set material requires focal attention. It should be emphasized, however, that the distinction between stimulus and response set is not an absolute one, and that in most real life situations the decision of whether a certain situation is of stimulus or response set nature-and accordingly whether preattentive or focal attention is the dominant process-should be made on a relative scale. Thus, for example, distinguishing between letters and digits, though mainly being a response set task, also has some stimulus set aspects, since some subtle physical feature differences between letters and digits may exist. Hence, the continuum between stimulus and response set material, and, correspondingly, the degree of involvement of preattentive processes and focal attention, suggests a similarity to "levels" of processing.

In the present experiment, an incidental learning paradigm was used with two different kinds of stimulus 
D

$\mathbf{N}$ 9

D

E

9

$\cdot \mathbf{G}$

G

2 3 5 $(\cdot)$

Figure 1. Example of a response set display: (a) grouped condition; (b) mixed condition.

material, stimulus and response set arrays. In the stimulus set conditions, subjects were presented with an array of digits and letters, half of the array containing red and the other half black characters, and were required to identify which one of two letters (digits) was presented. The color of the target was specified at the beginning of the experiment, and the subjects were instructed explicitly to attend to this color. In the response set condition, the subjects were presented with an array, half letters and half digits (all in black), and were also required to identify which of two letters (digits) was presented. In this condition, they were explicitly instructed to attend only to the letters (digits). Since response set material requires "deeper" processing (i.e., the use of focal attention), it was predicted that recall of the irrelevant material should be superior under response set than under stimulus set conditions. Neisser (1967) has emphasized the "constructive" activity of attention and claimed that focal attention requires a significantly larger amount of constructive effort compared with preattentive mechanisms which will result in a superior memory trace. Hence, it was predicted that memory for response set material would be better than for stimulus set material.

An additional variable, namely, the spatial arrangement of the stimuli, was also manipulated, to obtain further understanding of the nature of the two processes. More specifically, Neisser (1967) suggested that preattentive mechanisms may operate in parallel, while the processing of focal attention is serial in nature. Hence, it was predicted that, under stimulus set conditions, selection-which is made through preattentive processes-should be independent of the spatial arrangement of the array. For response set material, on the other hand, which is mainly handled by focal processes, selection can be made only after relatively detailed processing, and in such a case the spatial arrangement of the stimulus array should affect performance.

\section{METHOD}

\section{Subjects}

The subjects were 64 undergraduate students taking an introductory course in psychology at the University of Illinois. Participation partially fulfilled a course requirement. All had normal or corrected to normal vision.
Apparatus and Stimulus Material

A Scientific Prototype Model GA three field tachistoscope was employed with Sylvania F4T5/CWX lamps. Luminances of the three fields were set at $4.5 \mathrm{fl}$, as measured with a Spectra Spot photometer.

The stimuli consisted of the letters, $\mathrm{D}, \mathrm{H}$ (as targets) and $\mathrm{N}$, E, G, P (as background field) and the digits 4,8 (targets) and 2, 3, 5, 9(background field). These characters were either black or red, all taken from Letraset No. 47-18-CLN, .175 in. high and subtending $.18 \mathrm{deg}$ of visual angle. They were arranged on an imaginary circle which subtended 2 deg of visual angle in diameter. The circle was divided into eight positions (equally spaced), and the field items were positioned in seven of them, leaving one blank position for the target. The seven field characters were mounted on white vinyl plastic cards. Each target item was mounted on one of four separate clear plastic cards, each in a different position of the imaginary circle (corresponding to positions $1,3,5$, and 7 , going clockwise). Hence, every display presented to the subject consisted of a combination of a white and a clear plastic card.

For the response set condition, 16 target cards were prepared ( 4 cards for each of the four targets $D, H, 4,8$ ) and 16 background cards -8 cards with four letters and three digits, and 8 cards with four digits and three letters. Each set of 8 cards was again divided into two sets: In 4 cards, digits and letters were grouped; in the other four, letters and digits were mixed (see Figure 1). Thus there were four sets of background cards. Each set contained exactly the same characters except that the order of background stimuli and the position of the blank space for the target were changed.

The stimulus set cards were prepared in a similar manner, except that red and black served as an additional dimension. Therefore, an additional set of 16 target cards was prepared, identical to the former except for the color, which was red. Half of the background cards contained three red and four black characters (the empty position left for a red target), and the other half contained four red and three black characters (the empty position left for a black character).

Response sheets (identical for all conditions) were prepared. The digits $1,2,3,5,6,7,9$ and the letters $C, E, F, G, K, N, P$ were printed in two columns, and the subject had to circle the seven field characters which he remembered appearing on the display.

\section{Procedure}

There was a total of four conditions: Type of stimulus material (stimulus vs. response set) and spatial location of relevant and irrelevant set (mixed vs. grouped). The subjects were assigned randomly to each of the four groups (16 in each).

The subjects were told that they were going to participate in a visual perception experiment in which their task on each trial would be to identify which one of two possible targets, a D or an $H$ (4 or 8) was present among the eight characters of the visual display. In the response set condition, they were explicitly told to search among the letters-the relevant set-for a $D$ or an $H$ ( 4 or 8 for subjects where digits were the relevant set) and ignore the irrelevant set, i.e., the digits (letters). In the stimulus set condition they were told to search for a $D$ or an $H(4$ or 8$)$ among the relevant set, which consisted of black (red) letters and digits, and ignore the irrelevant set, i.e., the red (black) characters. Upon pressing a switch button, the fixation field (a black cross in the center of the visual field) disappeared and was replaced by a blank field, which lasted for $150 \mathrm{msec}$ and was followed by the stimulus display, which stayed on for $500 \mathrm{msec}$. After termination of the trial, the subject reported whether a D or an $\mathrm{H}(4$ or 8 ) was present, and the experimenter informed him whether the response was correct. The subjects were told to guess when they had not been able to detect the target.

After the subject received the instructions, and before he started the experimental trials, he was given three "dummy" presentations in which the display contained eight Os ordered in a circle in the same positions as the actual display. The pur- 
pose of this procedure was to introduce the subject to the pattern of the display and the exposure duration. These were followed by 32 experimental trials; each combination of target and background was presented four times during the experiment. The order of stimulus presentations was randomized.

The subject was given a response sheet immediately after his response to the last trial, and the experimenter read the following instructions (which also appeared written on the response sheet): "Each display that you have seen contained a target and 7 other characters which were the same through all the presentations. Please indicate below, by circling the appropriate characters, which were the 7 additional characters according to the best of your memory." The subjects were instructed to circle seven characters and to guess if they could not remember all of them. Notice, that under both conditions subjects did not know that half of the characters would be letters, the other half digits. They were only informed that the display would contain both let ters and digits.

\section{RESULTS}

Results are summarized in Tables 1-4. Table 1 presents means and standard deviations of the total number of correct characters (out of 7) identified on the recognition test. It can be seen that, as expected, memory is superior under response set compared with stimulus set conditions. An analysis of variance revealed that the difference in the number of correct identifications under these two conditions (stimulus vs. response set) was statistically significant, $F(1,60)=7.75, p<.01$. Twelve percent of the variance were accounted for by this effect. It is plausible to assume that the effect would have been larger except for guessing; the data reveals that standard deviations for the response set groups were much smaller. An $F$-test comparing the variances of the stimulus and response set groups yielded a significant difference, $p<.02$. This suggests that guessing probably occurred more often under stimulus set conditions. Both the spatial arrangement effect and the interaction were not significant.

Since the hypothesis predicts that there should be a memory difference only for the irrelevant sets, the data for the relevant and irrelevant sets were analyzed separately. Table 2 presents the means and standard deviations of correct identifications from the relevant set (the set to which the subject was instructed to attend). Three questions are of interest in this data: (1) Overall, is there a difference between stimulus set and response set conditions? (2) Within the stimulus set condition, does the spatial arrangement have any effect? (3) Within

Table 1

Means and Standard Deviations of the Total Number of Characters Correctly Recognized

\begin{tabular}{llcrr}
\hline & & $\begin{array}{c}\text { Stimulus } \\
\text { Set }\end{array}$ & $\begin{array}{c}\text { Response } \\
\text { Set }\end{array}$ \\
\hline \multirow{3}{*}{ Grouped } & Mean & 4.06 & 4.81 & 4.44 \\
& Mixed & 1.12 & .75 & 1.01 \\
& Mean & 4.37 & 4.94 & 4.66 \\
& SD & 1.09 & .77 & .97 \\
& Mean & 4.21 & 4.88 & 4.54 \\
& SD & 1.10 & .76 & .99 \\
\hline
\end{tabular}

Table 2

Means and Standard Deviations of Number of Characters Correctly Recognized from the Relevant Set

\begin{tabular}{llrrr}
\hline & & Stimulus & Response & \\
Set & & \multicolumn{1}{c}{ Set } & \\
\hline \multirow{3}{*}{ Mrouped } & Mean & 2.63 & 2.75 & 2.69 \\
& SD & .62 & .48 & .55 \\
& Mean & 2.75 & 2.31 & 2.53 \\
& SD & .58 & .60 & .62 \\
& Mean & 2.69 & 2.53 & 2.61 \\
& SD & .59 & .57 & .59 \\
\hline
\end{tabular}

Table 3

Means and Standard Deviations of Number of Characters Correctly Recognized from the Irrelevant Set

\begin{tabular}{|c|c|c|c|c|}
\hline & & $\begin{array}{c}\text { Stimulus } \\
\text { Set }\end{array}$ & $\begin{array}{c}\text { Response } \\
\text { Set }\end{array}$ & \\
\hline Grouped & $\begin{array}{l}\text { Mean } \\
\text { SD }\end{array}$ & $\begin{array}{r}1.44 \\
.96\end{array}$ & $\begin{array}{r}2.00 \\
.73\end{array}$ & $\begin{array}{r}1.72 \\
.88\end{array}$ \\
\hline \multirow[t]{2}{*}{ Mixed } & $\begin{array}{l}\text { Mean } \\
\text { SD }\end{array}$ & $\begin{array}{r}1.63 \\
.95\end{array}$ & $\begin{array}{r}2.63 \\
.62\end{array}$ & $\begin{array}{r}2.13 \\
.93\end{array}$ \\
\hline & $\begin{array}{l}\text { Mean } \\
\text { SD }\end{array}$ & $\begin{array}{r}1.53 \\
.94\end{array}$ & $\begin{array}{r}2.31 \\
.74\end{array}$ & $\begin{array}{r}1.92 \\
.93\end{array}$ \\
\hline
\end{tabular}

the response set condition, does the spatial arrangement have any effect? To answer the above questions, an analysis of preplanned comparisons was conducted, the three comparisons used corresponding to the above three questions. Only the third comparison (between the mixed and grouped conditions within response set) yielded a significant result, $F(1,60)=4.78, p<.05$, which accounted for $8 \%$ of the total variance.

A similar analysis was conducted on the number of correct identifications out of the irrelevant set (Table 3). ${ }^{1}$ The comparison between the stimulus and response set conditions was highly significant, $F(1,60)=14.14$, $p<.001$, and the amount of variance accounted for by this effect was $18 \%$. The comparison between the mixed and grouped arrangements under the response set condition was also significant, $F(1,60)=4.59, p<.05$, and accounted for $7 \%$ of the variance. The same comparison within the stimulus set condition was not significant. Notice, again, that the standard deviations for the stimulus set conditions were larger compared with response set. Although the difference did not reach significance, it was definitely in the expected direction, suggesting that subjects had to guess more often under stimulus set conditions.

Finally, an analysis of variance was conducted on the number of errors made during the orienting task (i.e., the number of incorrect identifications of the target-see Table 4). The difference between stimulus and response set conditions was highly significant, $F(1,60)=40.8, p<.0005$, and accounted for $36 \%$ of the variance. The spatial arrangement effect was also significant, $F(1,60)=9.54, p<.005$, and accounted for $10 \%$ of the variance. The interaction between type of 
Table 4

Means and Standard Deviations of Number of Errors During the Orienting Task

\begin{tabular}{llccc}
\hline & & $\begin{array}{c}\text { Stimulus } \\
\text { Set }\end{array}$ & $\begin{array}{c}\text { Response } \\
\text { Set }\end{array}$ & \\
\hline \multirow{3}{*}{ Grouped } & Mean & 1.06 & 4.44 & 2.75 \\
& SD & 1.34 & 3.35 & 3.04 \\
& Mean & 2.50 & 6.63 & 4.56 \\
& SD & 1.59 & 2.55 & 2.96 \\
& Mean & 1.78 & 5.53 & 3.66 \\
& SD & 1.62 & 3.13 & 3.11 \\
\hline
\end{tabular}

stimulus material and spatial arrangement was not significant.

\section{DISCUSSION}

The results strongly support the notion that stimulus and response set material require different levels of processing, which in turn affects memory performance. The number of characters recalled on the incidental task was significantly larger under response set conditions. The source of this difference is mainly based on the difference in the number of characters recalled from the irrelevant set (there was no difference between the two types of material in the number of recognitions from the relevant set). It thus seems plausible that the relevant set under stimulus set conditions appears to "stand out" and that partial selection (by preattentive mechanisms) is made at an initial stage of the perceptual process. In other words, under response set conditions, each character has to be processed via "focal attention" before a decision can be made. Hence, under response set conditions, stimuli from the irrelevant set receive "deeper" processing, and this produces superior performance on the recognition test.

Treisman (1964) conducted a series of experiments to investigate the monitoring and storage of the irrelevant message during selective attention to one of two dichotic speech messages. Briefly, her results indicated that "the storage survival-time is longer for analyzed than for unanalyzed material"; the degree to which the material was analyzed was dependent upon the nature of the stimulus material: whether it was, according to the present terminology, a stimulus set (e.g., difference in sounds) or a response set condition (where meaning of the message was important). Treisman's results are congruent with the ones obtained in the present study, thus extending our conclusions to the auditory modality as well.

Some evidence to support the argument that the relevant set "stands out" under stimulus set conditions is obtained from the data on the number of errors (detecting the target) during the orienting task. It is apparent that detecting the target under stimulus set conditions was considerably easier than under response-set conditions (see Table 4).
Additional information with regard to the nature of the two underlying processes can be obtained from the spatial arrangement variable which affects stimulus and response set differentially. The analyses for both the relevant and the irrelevant set indicated that, with stimulus set material, there was no difference between the grouped and mixed conditions. This further supports the notion that the relevant set under stimulus set conditions "stands out" regardless of the spatial arrangement. These results might also suggest that stimulus set material may be processed in parallel, which is consistent with Neisser's (1967) notion that preattentive mechanisms operate in parallel.

For the response set material, on the other hand, the spatial arrangement yielded a reliable effect. Under response set conditions, there is no immediate distinction between the relevant and irrelevant set, and each character has to be processed in relatively more detail. However, the reader is reminded that the distinction between stimulus and response set is not all or none. As mentioned earlier, some subtle shape differences between letters and digits do exist, and these differences are apparently more salient when letters or digits are grouped together rather than mixed. In other words, for response set under the grouped conditions, some weak stimulus set cues do exist. Therefore, it is easier to attend to the relevant set under the response set-grouped condition than to the mixed one. For the irrelevant set, on the other hand, the mixed condition requires serial scanning of the entire field, which explains the relatively high memory performance under mixed conditions. Notice that the above discussion assumes that response set material is processed serially. Again, such a notion would be congruent with Neisser's (1967) focal attention processes, which are serial in nature.

Bennett (Note 1) studied extensively the spatial effect in visual selective attention, and concluded that it appears that spatial configuration per se is not all that important. The more important influence on performance, according to Bennett, appears to be the targetbackground similarity. The results of the present study extend and modify this conslusion: The nature of stimulus material interacts with the spatial arrangement. $^{2}$

In conclusion, previous studies have demonstrated the effects of different levels of processing on memory, but most dealt with differential processing of response set material, that is, postperceptual analysis (e.g., comparing the effects of structural vs. semantic processing). The present study has demonstrated effects of different levels of processing which are closer to the sensory end of the processing continuum, thus supporting Craik and Lockhart's (1972) assertion that deeper levels of perceptual processing have the effect of increasing memory strength. Similarly, Neisser (1967) pointed out that focal attention processes require constructive activity (which preattentive mechanisms do not) and that 
traces of this constructive activity are stored in memory. The conslusions drawn in the present paper are consistent with Neisser's theoretical framework.

\section{REFERENCES}

Broadbent, D. E. Stimulus set and response set: Two kinds of selective attention. In D. I. Mostofsky (Ed.), Attention: Contemporary theories and analysis. New York: AppletonCentury-Crofts, 1970.

Broadbent. D. E. Decision and stress. London: Academic Press, 1971.

CRAIK, F. I. M., \& LockhaRT, R. S. Levels of processing: A framework for memory research. Journal of Verbal Learning and Verbal Behavior, 1972, 11, 671-684.

Craik. F. I. M., \& Tulving, E. Depth of processing and the retention of words in episodic memory. Journal of Experimental Psychology: General, 1975, 104, 268-294.

FrYKLUND. I. Effects of cued-set spatial arrangement and targetbackground similarity in the partial-report paradigm. Perception \& Psychophysics. 1975, 17, 375-386.

Hyde, T. S., \& Jenkins, J. J. The differential effects of incidental tasks on the organization of recall of a list of highly associated words. Joumal of Experimental Psychology, $1969,82,472-481$.

Johnston, C. D., \& Jenkins, J. J. Two more incidental tasks that differentially affect associative clustering in recall. Journal of Experimental Psychology, 1971, 89, 92-95.
KEREN. G. Somc considerations of two alleged kinds of selective attention. Journal of Experimental Psychology: General, in press. NeIsser, U. Cognitive psychology. New York: AppletonCentury-Crofts, 1967.

Treisman, A. Monitoring and storage of irrelevant messages in selective attention. Journal of Verbal Leaming and Verbal Behavior, 1964, 3, 449-459.

Tresselt, M. E., \& Mayzner, M. S. A study of incidental learning. Journal of Psychology, 1960, 50, 339.347.

\section{NOTES}

1. At first glance it might seem as if the number of errors for the stimulus set condition is less than chance. However, since the subjects did not know that there was an equal number of letters and digits in the display, it is impossible to compute any chance figures for the subanalyses.

2. Keren (1975) has shown that spatial arrangement and type of stimulus material strongly interact for any task which demands memory functions. It is less salient for simple search tasks, which is the reason why the interaction between spatial arrangement and stimulus material was not significant for the orienting task. In addition, the orienting task included only a small number of trials, the first few of which actually served as practice trials. Hence, any results derived from the orienting task should be taken with some caution.

(Received for publication November 14, 1975; revision received March 8, 1976.) 